\title{
Evaluation of estimated genetic values and their application to genome-wide investigation of systolic blood pressure
}

\author{
Ellen E Quillen ${ }^{1}$, V Saroja Voruganti ${ }^{1}$, Geetha Chittoor $^{1}$, Rohina Rubicz ${ }^{1}$, Juan M Peralta ${ }^{1,2}$, Marcio AA Almeida ${ }^{1}$, \\ Jack W Kent Jr', Vincent P Diego ${ }^{1}$, Thomas D Dyer ${ }^{1}$, Anthony G Comuzzie ${ }^{1}$, Harald HH Göring ${ }^{1}$, \\ Ravindranath Duggirala', Laura Almasy ${ }^{1}$, John Blangero ${ }^{* *}$
}

From Genetic Analysis Workshop 18

Stevenson, WA, USA. 13-17 October 2012

\begin{abstract}
The concept of breeding values, an individual's phenotypic deviation from the population mean as a result of the sum of the average effects of the genes they carry, is of great importance in livestock, aquaculture, and cash crop industries where emphasis is placed on an individual's potential to pass desirable phenotypes on to the next generation. As breeding or genetic values (as referred to here) cannot be measured directly, estimated genetic values (EGVs) are based on an individual's own phenotype, phenotype information from relatives, and, increasingly, genetic data. Because EGVs represent additive genetic variation, calculating EGVs in an extended human pedigree is expected to provide a more refined phenotype for genetic analyses. To test the utility of EGVs in genome-wide association, EGVs were calculated for 847 members of 20 extended Mexican American families based on 100 replicates of simulated systolic blood pressure. Calculations were performed in GAUSS to solve a variation on the standard Best Linear Unbiased Predictor (BLUP) mixed model equation with age, sex, and the first 3 principal components of sample-wide genetic variability as fixed effects and the EGV as a random effect distributed around the relationship matrix. Three methods of calculating kinship were considered: expected kinship from pedigree relationships, empirical kinship from common variants, and empirical kinship from both rare and common variants. Genome-wide association analysis was conducted on simulated phenotypes and EGVs using the additive measured genotype approach in the SOLAR software package. The EGV-based approach showed only minimal improvement in power to detect causative loci.
\end{abstract}

\section{Background}

Given increasing evidence that the majority of variation in common, complex traits is the result of a large number of individual variants with small effects, refining phenotypes to minimize the environmental component is one possible approach to increasing power to detect these variants. This work extends a common concept in plant and animal breeding, the estimated breeding value, to calculate estimated genetic values (EGVs) in human

\footnotetext{
* Correspondence: john@txbiomedgenetics.org

'Department of Genetics, Texas Biomedical Research Institute, PO Box 760549, San Antonio, TX 78245, USA

Full list of author information is available at the end of the article
}

pedigrees. The breeding value is the deviation of an individual's phenotype from the population mean as a result of the sum of the average effects of the genes they carry. There are several methods for estimating breeding values, with the Best Linear Unbiased Prediction (BLUP) used most frequently. In its most basic form, BLUP accounts for additive genetic and environmental covariances among relatives based on known pedigree structure. Several extensions of BLUP have been developed to calculate the genomic estimated breeding values, which are derived directly from molecular genetic information and are commonly used for genomic selection in plant and animal breeding programs [1,2]. The accuracy 
of and variation in EGVs are increased when phenotype heritability estimates are higher and by the inclusion of additional information from relatives, so the method is most useful in extended pedigrees. Because EGVs represent predominantly additive genetic variance (some additional environmental variance may be included where it mimics relatedness), the use of EGVs in place of standard phenotypes will increase heritability and may also increase power to detect variants of smaller effect. Although high heritability does not guarantee the identification of causal variants [3], it is one possibility out of a variety of methods to increase the identification of variants that frequently fall below the significance threshold in genome-wide association (GWA) studies. Zabaneh and Mackay [4] examined the suitability of using pedigree-based EGVs in genome-wide linkage analysis, and found that this method improves power to detect quantitative trait loci. However, because EGVs are a product of genetic similarities of individuals in the sample, the use of empirically derived relationship matrices in calculating EGVs should increase power to localize genetic factors in genome-wide association (GWA) studies, an observation that has driven the use of relationship matrices in artificial selection [[5] and others].

\section{Methods}

\section{Sample description}

To determine the suitability of EGVs for human quantitative traits, the simulated visit 1 systolic blood pressure (SBP) values from the Genetic Analysis Workshop 18 (GAW18) data set were considered [6]. R princomp [7] was used to perform principal components analysis on the 117 unrelated individuals in the sample using a subset of 28,157 single-nucleotide polymorphisms (SNPs) selected for uniform coverage and low mutual linkage disequilibrium (LD) from the SNPs provided. The resulting principal component $(\mathrm{PC})$ scores were projected on the full set of related individuals by assigning offspring the mean of parental scores. Using SOLAR [8], residual SBP values for each simulation were obtained from fitting a polygenic model incorporating age, sex, and the first three PCs as covariates. The value of these covariates remained the same across all simulations, but their effects on SBP varied. Expected relatedness (2Ф) based on the provided pedigree was calculated in SOLAR. Additionally, empirical relatedness was calculated in KING [9], based on either common variants (472,050 SNPs located on odd-numbered chromosomes) or all variants extracted from the sequence data on oddnumbered chromosomes. Of the 2 methods offered in KING, the robust method was selected as it employs a family-specific correction for substructure.

\section{EGV calculation}

The residualized SBP values and relationship matrices, $\mathbf{R}$, equal to $2 \Phi$ were used in calculating EGVs. Throughout, EGVs will be subscripted with the origin of the $\mathbf{R}$ matrix so that $E G V_{\text {ped }}$ is derived from the expected, pedigree-based matrix, $\mathrm{EGV}_{\text {snp }}$ from the empirical SNPbased matrix, and $\mathrm{EGV}_{\text {seq }}$ from the full sequence-based matrix. EGVs were calculated from a variation of the standard BLUP estimation of breeding values using a custom script written in GAUSS (Aptech Systems, Inc.), which is available upon request. At the core of the calculation is the mixed model

$$
E G V=\left(I+R^{-1} \frac{\sigma_{e}^{2}}{\sigma_{g}^{2}}\right)^{-1}(\gamma-X \beta)
$$

where $X$ is the matrix of fixed effects (sex, age, and the first $3 \mathrm{PCs}$ ) included as covariates in the polygenic analysis, $\gamma$ is the observed value of SBP, and $\beta$ is the fixed-effects coefficient. In full, $\gamma-X \beta$ is the residual values derived from the variance component model discussed above. The additive genetic variance $\left(\sigma_{g}^{2}\right)$ and environmental variance $\left(\sigma_{e}^{2}\right)$ are also obtained from the polygenic model fit in SOLAR. Because the $\mathbf{R}$ matrices produced by KING are not positive, semidefinite-a requirement for solving the model-the nearest correlation matrix was calculated by the alternating projections procedure described by Higham [10] and implemented in GAUSS [11].

\section{Genome-wide association}

GWA was performed in SOLAR for the raw SBP values, $E G V_{\text {ped }}, E V_{\text {snp }}$, and $\mathrm{EGV}_{\text {seq. }}$ Although EGVs can be estimated for unphenotyped individuals, sample size remained the same across all tests as the simulated data set has phenotype and genotype information for all 847 nonidentical individuals.

GWA was performed using the measured genotype association (MGA) test, which applies a likelihood ratio test to an additive model of allelic effects while including a covariance matrix of expected pairwise relatedness to control for kinship. The pairwise error variance matrix calculated in the GAUSS script was also incorporated into the MGA model such that

$$
\Omega=\sigma_{p}^{2}\left(2 \Phi h^{2}+I \sigma_{e}^{2}+E \varepsilon\right)
$$

where $\Omega$ is the phenotypic covariance matrix, $\Phi$ is the expected kinship matrix, $h^{2}$ is narrow-sense heritability, I is the identity matrix, $\sigma_{p}^{2}$ and $\sigma_{e}^{2}$ are phenotypic and environmental variance components, $\mathbf{E}$ is the error variance matrix, and $\varepsilon$ is the corresponding error parameter. Under the MGA test, the log-likelihood of the 
null model with no SNP effect is compared to the loglikelihood of the alternative model with an estimated SNP effect. The likelihood ratio test, given as minus twice the difference of the null and alternative log-likelihoods, is distributed as a chi-square variate with 1 degree of freedom.

A major advantage of the simulated data is the ability to determine the precise false-discovery rate of the method. For each of the top 55 variants contributing to simulated SBP and all SNPs identified in the GWA analyses $\left(p<5 \times 10^{-7}\right)$, pairwise correlations $\left(\mathrm{R}^{2}\right)$ were calculated in SOLAR to assess the extent of local LD. An associated SNP was considered accurately identified if it fell within an LD block defined by $\mathrm{R}^{2} \geq 0.2$ surrounding a simulated causative gene; otherwise the associated SNP was deemed a false positive.

\section{Results}

\section{Expected and empirical relatedness}

The use of empirical relatedness measures is increasing in popularity as a means of resolving the variation around pedigree-based relatedness estimates and accounting for cryptic relatedness and inbreeding. These factors will increase mean relatedness in a population, as reflected in the larger SNP-based and sequence-based pairwise kinships relative to the pedigree-based values. In all cases, the heritability of the EGVs is approximately double the heritability of simulated SBP with covariates included. This is expected as the computation of EGVs removes much of the environmental variation seen in SBP. However, this also indicates that not all nongenetic variation has been removed.

\section{GWA results from EGVs}

There is broad variation in the number of significantly associated SNPs $\left(p<5 \times 10^{-8}\right)$ across the simulations and within or among methods. On average, $\mathrm{EGV}_{\text {snp }}$ and $E_{\text {Seq }}$ identified more SNPs than the raw SBP GWA, but the median number of associations was approximately the same (Table 1). The simulation was designed so that 15 SNPs in MAP4 comprise $7.79 \%$ of the variation in SBP, 14 SNPs in NRF1 4.67\%, 16 SNPs in TNN $3.87 \%$, and 8 SNPs in LEPR 2.06\%. Additional genes had extremely small effects. Table 2 shows the number of simulations in which each expected gene was identified for each method. All methods reliably identified SNPs in MAP4 and neighboring gene DNASE1L3, with additional SNPs in BTD and SUMF1 also associated because of the extended LD and the large effect of MAP4. SNPs in LEPR were only identified in $4 \%$ to $7 \%$ of cases, with no method clearly outperforming the others. MAP4 and LEPR each contained a single SNP contributing more than $2 \%$ of the variance, which is likely why no SNPs in NRF1 or TNN were identified despite these genes contributing more to SBP in their entirety. Among the genes that explain less than $2 \%$ of the overall variance, none was identified by any method in more than $5 \%$ of trials.

Of particular interest for complex traits like SBP is the effect of rare variants, a feature reflected in the design of the simulation with 10 of the top 55 causal variants present at minor allele frequency (MAF) less than $1 \%$ and 28 at less than $5 \%$. In contrast, less than $12 \%$ of significantly associated SNPs identified by any method have a MAF $\leq 0.05$ and only $1 \%$ have a MAF $\leq 0.01$.

Overall, the false-discovery rate (FDR) is low, with FDR approximately stable across the raw SBP and EGV GWAs (see Table 1), and little evidence of inflation in lambda values. However, as this method aims to move contributing variants out of the suggestively associated "gray zone" without inflating type II error, the error rate was also calculated specifically for SNPs associated with the EGV values, but not the raw SBP values. These

Table 1 Description of GWA results for EGVs and SBP across simulations.

\begin{tabular}{|c|c|c|c|c|}
\hline & SBP & $\mathrm{EGV}_{\text {ped }}$ & $\mathrm{EGV}_{\text {snp }}$ & $\mathrm{EGV}_{\text {seq }}$ \\
\hline Total number of significant (sig) SNPs in 100 simulations & 166 & 134 & 191 & 273 \\
\hline Sig SNPs with minor allele frequency (MAF) $<0.05$ & $12.0 \%$ & $5.2 \%$ & $11.5 \%$ & $6.6 \%$ \\
\hline Sig SNPs with MAF $<0.01$ & $1.2 \%$ & $0.7 \%$ & $1.0 \%$ & $0.7 \%$ \\
\hline Mean number of sig SNPs & 11.9 & 7.8 & 13.9 & 14.1 \\
\hline Median number of sig SNPs & 8 & 2 & 8 & 8 \\
\hline stdev in number of sig SNPs & 15.8 & 15.0 & 18.4 & 19.3 \\
\hline False-discovery rate (FDR) & $7.7 \%$ & $5.7 \%$ & $6.7 \%$ & $7.7 \%$ \\
\hline FDR for SNPs not seen in SBP & - & $52.1 \%$ & $50.0 \%$ & $40.4 \%$ \\
\hline Smaller average $p$ value for sig SNPs & - & $30.0 \%$ & $57.0 \%$ & $48.0 \%$ \\
\hline Simulations identifying more SNPs than SBP & - & 14 & 39 & 36 \\
\hline Simulations identifying fewer SNPs than SBP & - & 77 & 18 & 30 \\
\hline Simulations identifying same number of SNPs as SBP & - & 9 & 43 & 34 \\
\hline
\end{tabular}

For 100 replicates of simulated SBP, GWA was performed on the raw data, $\mathrm{EGV}_{\text {ped, }} \mathrm{EGV}_{\text {snp, }}$ and $\mathrm{EGV}_{\text {seq. }}$ The following table gives descriptive statistics for significant SNPs $\left(p<5 \times 10^{-8}\right)$ by method. The last five rows illustrate the performance of the EGV method relative to the raw SBP GWA. 
Table 2 Significant results for GWA of EGVs and SBP by gene.

\begin{tabular}{cccccc}
\hline Gene & Chr & SBP & EGV $_{\text {ped }}$ & EGV $_{\text {snp }}$ & EGV $_{\text {seq }}$ \\
\hline LEPR & 1 & 7 & 4 & 6 & 4 \\
LRP8 & 1 & 6 & 0 & 8 & 5 \\
NEXN & 1 & 1 & 1 & 2 & 1 \\
BTD & 3 & 16 & 16 & 22 & 20 \\
DNASE1L3 & 3 & 100 & 95 & 100 & 99 \\
MAP4 & 3 & 100 & 95 & 100 & 99 \\
SUMF1 & 3 & 33 & 14 & 34 & 39 \\
MTRR & 5 & 3 & 0 & 3 & 4 \\
RHOD & 11 & 2 & 0 & 1 & 1 \\
TCIRG1 & 11 & 2 & 1 & 1 & 1 \\
CYP1A2 & 15 & 0 & 0 & 1 & 0 \\
C1QBP & 17 & 0 & 1 & 0 & 1 \\
KRT23 & 17 & 0 & 1 & 0 & 1 \\
RAI1 & 17 & 0 & 1 & 0 & 1 \\
SAT2 & 17 & 0 & 0 & 1 & 1 \\
COL5A3 & 19 & 0 & 1 & 1 & 0 \\
\hline
\end{tabular}

For each gene contributing to simulated SBP, the table lists the number of replicates (out of 100) in which at least one significant association was found. Variants in 24 additional genes have small effects on SBP but were never detected and were omitted from the table. Due to extended linkage disequilibrium, more than one gene may be tagged by a single variant; in particular, the associations in DNASE1L3 are likely due to strong LD with major causative gene MAP4.

FDRs were greatly inflated, with approximately half of these associations representing false positives (see Table 1).

\section{Discussion}

There are several measures of the utility of a novel method of GWA: strength of associations, absolute number of significant associations, number of genes or genomic regions identified, and the minimization of type I and type II error rates. Strength of association and number of significant associations will be strongly correlated and were expected to be the predominant avenue of improvement in the use of EGVs. Ideally, the use of EGVs would eliminate the influence of environmental variation and drive more causative SNPs from the "suggestive" into the "significant" association range of $p$ values. Although the use of EGVs does increase heritability by removing environmental variation and capturing only additive genetic variation, it does not guarantee a sufficient increase in power to detect extremely rare variants or deal with phenotypes with a very large number of causative genes (e.g., height). Based on these simulations, where many alleles with a low MAF and small effect sizes contribute to SBP, this method does not substantially increase the power to detect rare variants. This method is most likely to improve power in studies of large pedigrees where individuals have many close relatives, which maximizes accuracy of EGV calculation, in phenotypes with significant but difficult-to-quantify environmental components that can be removed by the EGV method, and where the majority of genetic variation is a result of additive effects.

\section{Conclusions}

The $\mathrm{EGV}_{\text {snp }}$ and $\mathrm{EGV}_{\text {seq }}$ methods, which employ empirical kinship estimates, slightly outperformed the standard MGA method based on the average number of truly causal SNPs identified. However, when judged on the basis of additional causal genes identified, the improvements are sporadic and fail to recognize genes of medium effect. Overall, the use of EGVs neither significantly increased nor decreased the ability to detect rare causal variants of small to modest effect.

\section{Competing interests}

The authors declare that they have no competing interests.

\section{Authors' contributions}

JB designed the overall study and assisted in statistical analyses. EEQ conducted the statistical analyses and drafted the manuscript; VSV, GC, and RR helped in statistical analyses and drafting the manuscript. JMP, MAAA, JWK, VPD, and TDD contributed additional data for the analyses. RD, HHHG, $L A$, and AGC contributed analytical oversight and comments. All authors approved the final manuscript.

\section{Acknowledgements}

The GAW18 whole genome sequence data were provided by the T2DGENES Consortium, which is supported by NIH grants U01 DK085524, U01 DK085584, U01 DK085501, U01 DK085526, and U01 DK085545. The other genetic and phenotypic data for GAW18 were provided by the San Antonio Family Heart Study and San Antonio Family Diabetes/Gallbladder Study, which are supported by NIH grants P01 HL045222, R01 DK047482, and R01 DK053889. The Genetic Analysis Workshop is supported by NIH grant R01 GM031575.

The authors are supported in part by NIH grants funding the T2D-GENE Consortium: U01 DK085524, U01 DK085501, U01 DK085526, U01 DK085584 and U01 DK085545; the SAFHS: P01 HL045222; the SAFDS: R01 DK047482; and the SAFGS: R01 DK053889. SOLAR is supported by NIMH grant MH059490 and the supercomputing facilities used for this work at the AT\&T Genetics Computing Center were supported in part by a gift from the SBC Foundation.

This article has been published as part of BMC Proceedings Volume 8 Supplement 1, 2014: Genetic Analysis Workshop 18. The full contents of the supplement are available online at http://www.biomedcentral.com/bmcproc/ supplements/8/S1. Publication charges for this supplement were funded by the Texas Biomedical Research Institute.

\section{Authors' details}

${ }^{1}$ Department of Genetics, Texas Biomedical Research Institute, PO Box 760549, San Antonio, TX 78245, USA. ${ }^{2}$ Centre for Genetic Origins of Health and Disease, University of Western Australia, 35 Stirling Hightway, Crawley, Western Australia, 6009, Australia.

Published: 17 June 2014

\section{References}

1. Kemper KE, Bowman PJ, Pryce JE, Hayes BJ, Goddard ME: Long-term selection strategies for complex traits using high-density genetic markers. J Dairy Sci 2012, 95:4646-4656.

2. Koivula M, Strandén I, Su G, Mäntysaari EA: Different methods to calculate genomic predictions-comparisons of BLUP at the single nucleotide polymorphism level (SNP-BLUP), BLUP at the individual level (G-BLUP), and the one-step approach (H-BLUP). J Dairy Sci 2012, 95:4065-4073. 
3. Manolio T, Collins FS, Cox NJ, Goldstein DB, Hindorff L, Hunter DJ, McCarthy MI, Ramos EM, Cardon LR, Chakravarti A, et al: Finding the missing heritability of complex diseases. Nature 2009, 461:747-753.

4. Zabaneh D, Mackay IJ: Genome-wide linkage scan on estimated breeding values for a quantitative trait. BMC Genet 2003, 4:1-6.

5. Hayes BJ, Visscher PM, Goddard ME: Increased accuracy of artificial selection by using the realized relationship matrix. Genet Res 2009, 91:47-60.

6. Almasy L, Dyer TD, Peralta JM, Jun G, Fuchsberger C, Almeida MA, Kent JW Jr, Fowler S, Duggirala R, Blangero J: Data for Genetic Analysis Workshop 18: Human whole genome sequence, blood pressure, and simulated phenotypes in extended pedigrees. BMC Proc 2014, 8(suppl 2):S2.

7. R Development Core Team RFFSC: R: A Language and Environment for Statistical Computing. Vienna Austria R Foundation for Statistical Computing 2008, 1.

8. Almasy L, Blangero J: Multipoint quantitative-trait linkage analysis in general pedigrees. Am J Hum Genet 1998, 62:1198-1211.

9. Manichaikul A, Mychaleckyj JC, Rich SS, Daly K, Sale M, Chen W-M: Robust relationship inference in genome-wide association studies. Bioinformatics 2010, 26:2867-2873.

10. Higham NJ: Computing the nearest correlation matrix-a problem from finance. IMA J Numer Anal 2002, 22:329-343.

11. Rapuch G, Roncalli T: GAUSS Procedures for Computing the Nearest Correlation Matrix and Simulating Correlation Matrices. Groupe de Recherche Opérationnelle Credit Lyonnaise, Lyon, France; 2001, 1-25.

doi:10.1186/1753-6561-8-S1-S66

Cite this article as: Quillen et al.: Evaluation of estimated genetic values and their application to genome-wide investigation of systolic blood pressure. BMC Proceedings 2014 8(Suppl 1):S66.

\section{Submit your next manuscript to BioMed Central} and take full advantage of:

- Convenient online submission

- Thorough peer review

- No space constraints or color figure charges

- Immediate publication on acceptance

- Inclusion in PubMed, CAS, Scopus and Google Scholar

- Research which is freely available for redistribution

Submit your manuscript at www.biomedcentral.com/submit
Ciomed Central 